\title{
Measuring the Street Layout Orientation Entropy of Cities in the Philippines
}

\author{
Homer Pagkalinawan ${ }^{\mathrm{a}, \mathrm{b}}$ * \\ ${ }^{a}$ COBENA Business Analytics and Strategy, Inc., homer.pagkalinawan@ gmail.com \\ ${ }^{b}$ Asian Development Bank \\ * Corresponding author
}

Keywords: Street Orientation, Entropy, Urban Environment, Socio-economics

\begin{abstract}
:
Selected cities in the Philippines follow, to a certain extent, an organized street layout and orientation based on a designed plan including major cities like Manila and Quezon City. However, others cities developed organically on a less organized manner, expanding haphazardly. Being the spatial backbone of these cities, street patterns controls and limits the flow of people, goods, and activities. Measuring entropy, or the state of orderliness or disorderliness, of a street network can assess the difference between a planned and an unplanned city development. Possibly, it can be correlated to the various socioeconomic variables e.g. population, density, income level, poverty level, etc. or environmental variables e.g. night time light data, urban heat, vegetation cover, etc., that characterizes a city.
\end{abstract}

Entropy values and measure of grid orderliness was computed for all cities and municipalities in the Philippines. Shannon entropy equation (Figure 1) was used to measure the distribution of a city's street layout orientation.

$H_{x}=-\sum_{i}^{n} P\left(x_{i}\right) \ln P\left(x_{i}\right)$

where $\mathrm{H}_{\mathrm{x}}$ is the entropy value and $\mathrm{P}\left(\mathrm{x}_{\mathrm{i}}\right)$ is the proportion of distribution of the $\mathrm{i}^{\text {th }}$ bin

Figure 1. Shannon entropy equation

Using entropy value $\left(\mathrm{H}_{\mathrm{x}}\right)$, a measure of grid-orderliness (Figure 2) was also calculated. $\mathrm{H}_{\max }$ or the maximum entropy was computed when a city's street orientation is perfectly distributed across all directions while $\mathrm{H}_{\text {grid }}$ is the entropy value when a city's street orientation is an ideal grid street plan. A value of 0 indicates a maximum entropy or perfect disorder among the city's street layout while a value of 1 .

$\rho_{\text {city }}=\left|1-\left(\frac{H_{x}-H_{\text {grid }}}{H_{\text {max }}-H_{\text {grid }}}\right)^{2}\right|$

where $\rho_{\text {city }}$ is the grid orderliness measure

Figure 2. Shannon entropy equation

All 136 Philippines cities were evaluated for their grid orderliness. Seven of the top 10 cities in term of grid orderliness were located in Mindanao Island - Tacurong, Sultan Kudarat (1), Tangub, Misamis Occidental (2), Koronadal, South Cotabato (3), General Santos, Sarangani (4), Digos, Davao del Sur (5), Dipolog, Zamboanga Del Norte (6), and Kidapawan, North Cotabato (10). Most areas in the Mindanao Island were developed during the American occupation of the Philippines, calling it the "Land of Promise" and building these new city settlements with gridded streets. Figure 3 shows the location of these cities in Mindanao Island as well as the street lay-out and street direction for Tacurong City, the Philippine city with the higher grid orderliness. 


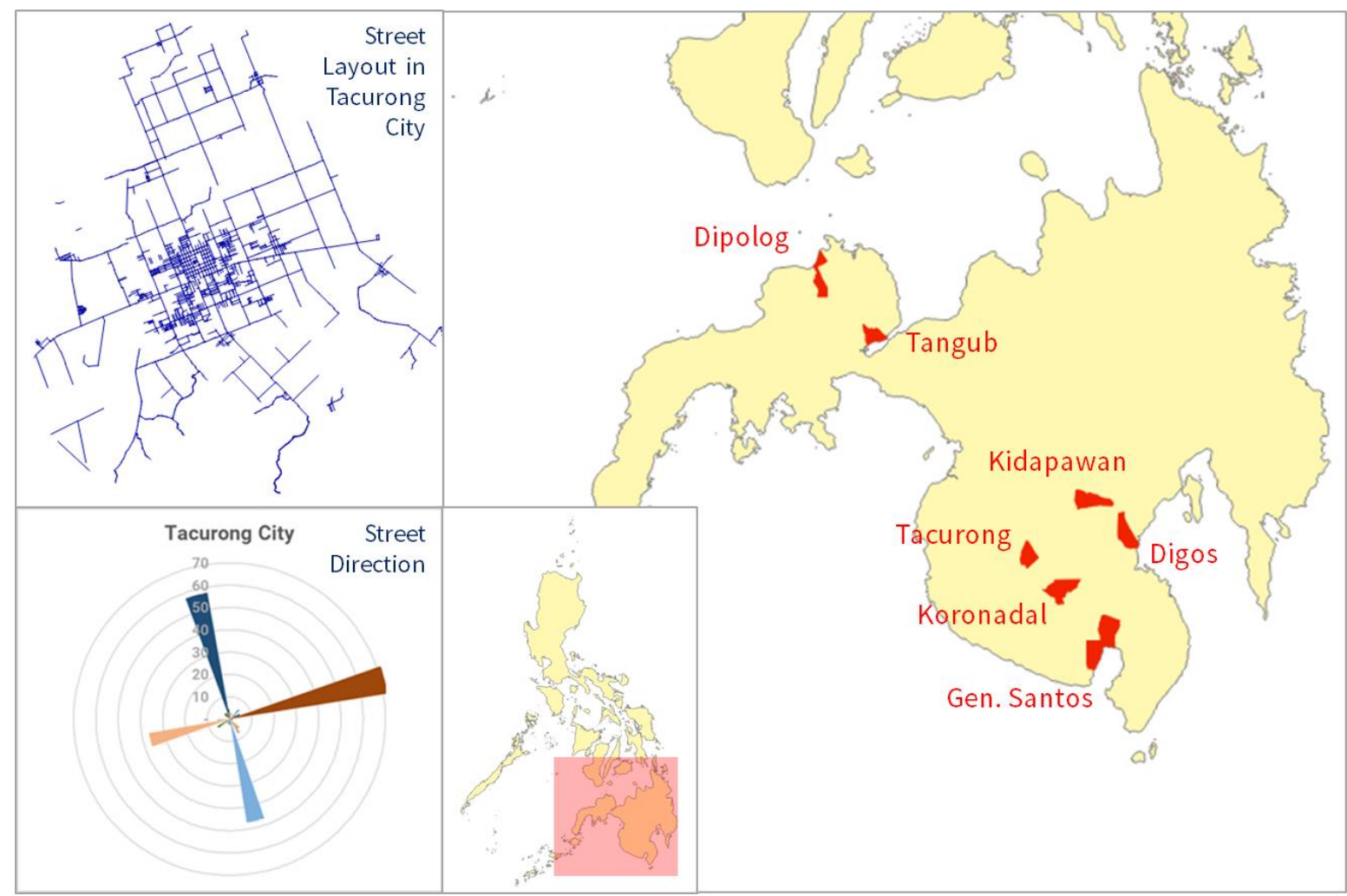

Figure 3. Street Layout in Tacurong City and Street Direction Graph for Tacurong City (left); Top Cities in Terms of Grid Orderliness (right).

Looking at the country's capital, Metropolitan Manila, and its seventeen (17) local government units, two north-south directions dominated the street direction graph (see Figure 4). These directions coincide with the elongated shape of the capital, as it is constrained from developing longitudinally due to presence of bodies of water from both sides. Unlike cities in Mindanao which were developed using gridded patterns, streets in Metro Manila were limited by terrain constraints. The same case can be observed in cities located in higher elevation such as Baguio and Passi.

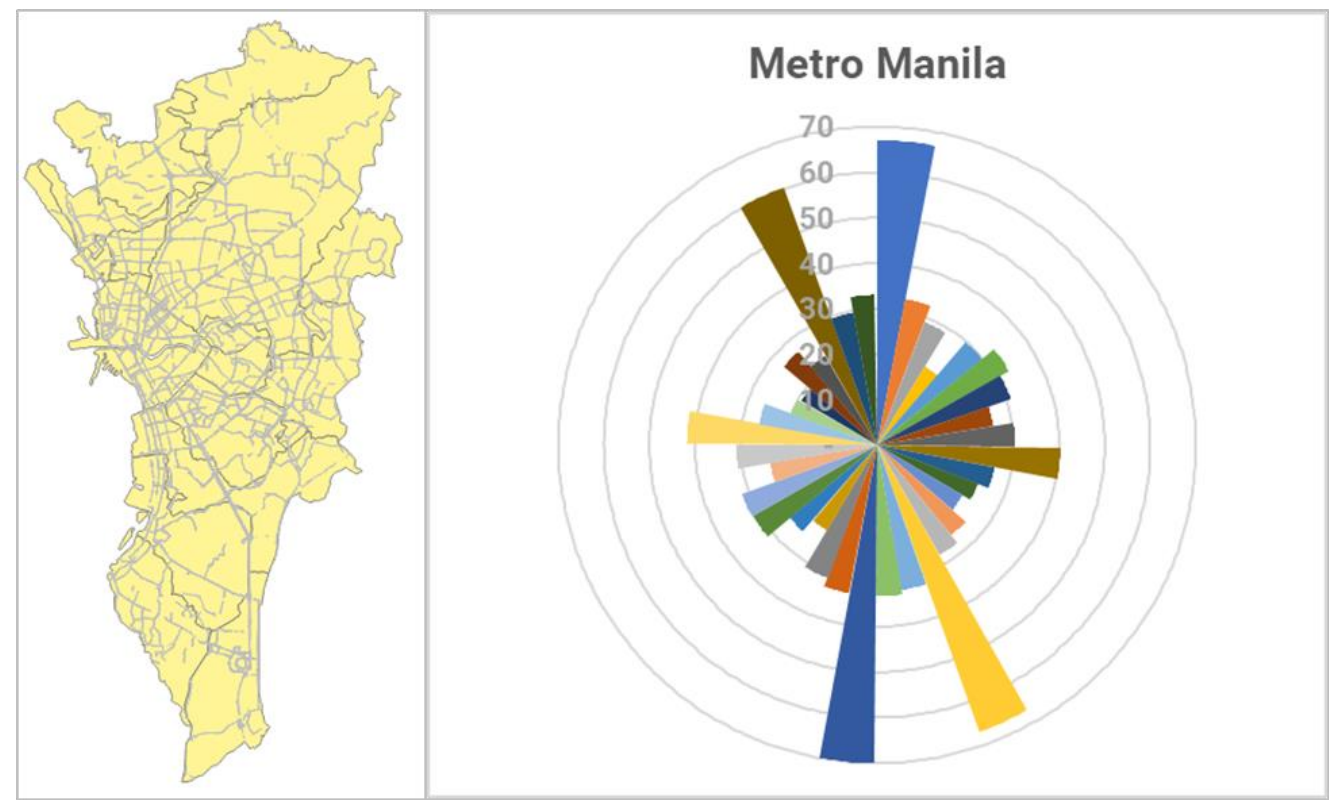

Figure 4. Street Map of Metropolitan Manila and corresponding Street Direction Graph.

\section{References:}

Boeing, Geoff. 2018. Urban Spatial Order: Street Network Orientation, Configuration, and Entropy. DOI: arXiv:1808.00600v2 